\title{
Author Correction: Diversity-dependent temporal divergence of ecosystem functioning in experimental ecosystems
}

Nathaly R. Guerrero-Ramírez DD, Dylan Craven (D), Peter B. Reich, John J. Ewel, Forest Isbell (D), Julia Koricheva, John A. Parrotta, Harald Auge (D), Heather E. Erickson, David I. Forrester, Andy Hector, Jasmin Joshi, Florencia Montagnini, Cecilia Palmborg, Daniel Piotto, Catherine Potvin, Christiane Roscher, Jasper van Ruijven, David Tilman, Brian Wilsey and Nico Eisenhauer (iD

Correction to: Nature Ecology \& Evolution https://doi.org/10.1038/s41559-017-0325-1 (2017), published online 2 October 2017.

In the version of this Brief Communication originally published, periodic annual increment of basal area and total basal area were calculated for Washington State 1 and 2 sites using a plot area of 0.4 ha rather than 0.1764 ha, which is the area in which trees were measured, and excludes buffer rows. This difference results in slight changes to the caption of Fig. 1; specifically: "For forest, the periodic annual increment of the basal area was significantly affected by species richness (old version: $F_{1,1433.1}=10.07, P<0.01$, corrected version: $F_{1,1432.9}$ $=9.98, P<0.01$ ) and the total basal area was significantly affected by time (old version: $F_{1,291.9}=24.32, P<0.001$, corrected version: $\left.F_{1,276}=31.14, P<0.001\right)$ and the species richness $\times$ time interaction (old version: $F_{1,291.9}=18.39, P<0.001$, corrected version: $F_{1,276}$ $=16.37, P<0.001)$. In Supplementary Table 2, there were slight changes to the model for the periodic annual increment of basal fixed effects and variance components (study and plot). In Supplementary Table 4, there were slight changes to the model for total basal area fixed effects, the variance components (study, study $\times$ species richness, study $\times$ species richness $\times$ time) and temporal autocorrelation. In Supplementary Figs 4 and 5 the panels with data from Washington State 1 and 2 were modified reflecting the changes to periodic annual increment of basal area and total basal area. All these errors have now been corrected; the amended panels from Supplementary Figs 4 and 5 are shown below. These changes did not affect the results nor the conclusions of the paper.

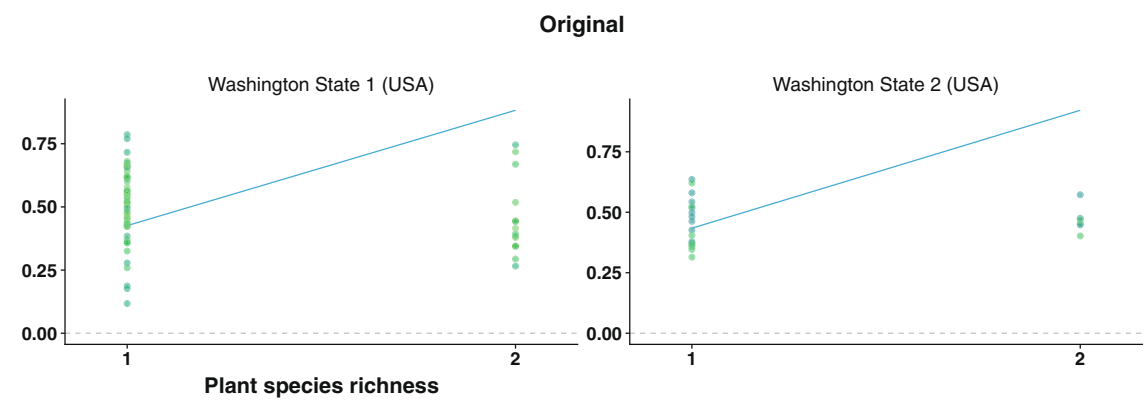

Corrected

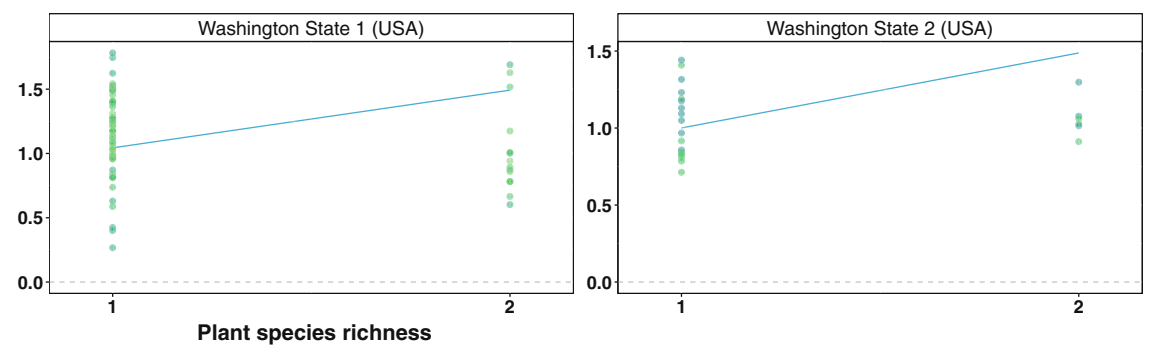

Supplementary Fig. 4 | Original and corrected panels for Washington State 1 and Washington State 2. 


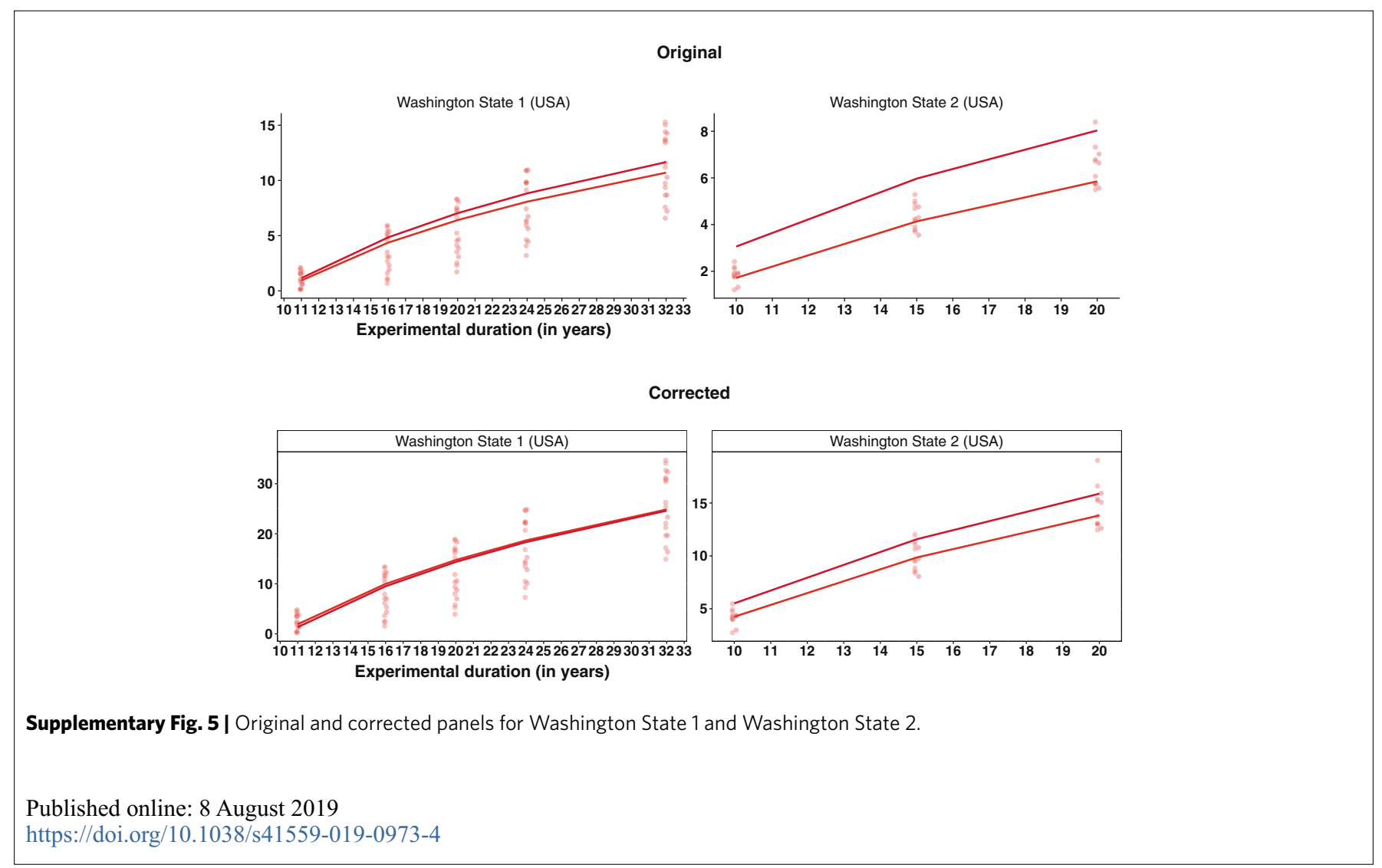

